\title{
Relaciones entre la microestructura y las propiedades mecánicas en fundiciones nodulares: revisión bibliográfica( ${ }^{(\cdot)}$
}

\author{
R. Castillo*, V. Bermont* y V. Martínez ${ }^{* *}$ \\ Resumen En este trabajo se revisan los avances realizados en el conocimiento de las relaciones entre \\ la microestructura y las propiedades mecánicas de las fundiciones nodulares. Se consideran \\ las aplicaciones de los tratamientos térmicos de austemperizado o temple bainítico que han \\ permitido mejorar sustancialmente las propiedades mecánicas de estas fundiciones (ADI). \\ Se propone un programa de investigación para la caracterización de la resistencia al \\ crecimiento de grietas bajo corrosión y modelización de las propiedades mecánicas.
}

Palabras clave Fundiciones nodulares. ADI. Propiedades mecánicas, Microestructura. Tratamientos térmicos. Revisión bibliográfica.

\section{Relationships between microstructure and mechanical properties in ductile cast irons: a review}

\begin{abstract}
The progress achieved in the understanding of the relationships between the microstructure and the mechanical properties of ductile cast iron is reviewed. It is also described the applications of heat treatment of austempered to ductile irons (ADI), which have allowed to improve substantially the mechanical properties of these materials. It is proposed a research program to obtain the crack growth resistance under corrosive atmospheres and to model the mechanical properties.
\end{abstract}

Keywords Ductile cast iron. ADI. Mechanical properties. Microstructure. Heat treatments. Review.

\section{INTRODUCCIÓN}

Dentro de los materiales ferrosos, las fundiciones con grafito libre tienen un extenso campo de aplicación. A pesar del avance relativo de otros materiales, algunas fundiciones han continuado ampliando su espectro de aplicaciones debido al mejoramiento de las propiedades mecánicas ${ }^{[1]}$, la facilidad de obtención y el bajo costo en relación con materiales tradicionales como el acero ${ }^{[2]}$.

El grafito libre otorga a las fundiciones propiedades tales como alta conductividad térmica, autolubricación, amortiguación de las vibraciones mecánicas, etc. La principal desventaja es que, por constituir el grafito una fase de resistencia mecánica nula, su presencia puede asemejarse a la de fisuras o huecos distribuidos por toda la pieza. Esto se traduce en una menor resistencia mecánica. El grafito libre se puede presentar en varias morfologías: laminar (fundición gris), esferoidal (fundición no- dular) o intermedia (fundición vermicular). Tradicionalmente la producción de hierro fundido estaba constituida por fundición gris con grafito laminar. Sin embargo, a partir de mediados de siglo se logró obtener tecnológicamente la morfología nodular $^{[2]}$. De esta forma pudo alcanzarse una mayor resistencia mecánica y ductilidad, debido a que esta morfología reduce el efecto de entalla de la fase grafito. Este tipo de fundición se llama fundición nodular, esferoidal o fundición dúctil.

El control de la morfología del grafito y del tamaño de célula, unido a la posibilidad de adecuar convenientemente la microestructura de la matriz mediante tratamientos térmicos, ha permitido un constante crecimiento de las aplicaciones industriales de las fundiciones, llegando a sustituir a los aceros aleados de distintos tipos, fundidos y forjados. Este es el caso particular de las fundiciones nodulares austemperizadas (austempered) ADI, las cuales pueden satisfacer los requerimientos de

(•) Trabajo recibido el 5 de marzo de 1999 y aceptado en su versión final el día 27 de septiembre de 1999.

$(*)$ Dpto. de Ingeniería Mecánica, Universidad de La Serena. Benavente, 980. La Serena (Chile).

$\left(^{* *}\right.$ Dpto. de Ingeniería Metalúrgica, Universidad de Santiago. Av. B. O’Higgins, 3363. Santiago (Chile). 
propiedades mecánicas de forma más económica ${ }^{[3]}$.

Las fundiciones nodulares austemperizadas ADI se obtienen por medio de un tratamiento térmico de transformación isotérmica de la austenita. Este consta de dos partes: austenización a $900{ }^{\circ} \mathrm{C}$ por una o más horas y temple hasta el rango de $450^{\circ} \mathrm{C}$ a $250^{\circ} \mathrm{C}$. Se debe mantener la temperatura para obtener la transformación bainítica. La matriz ADI está formada por ferrita acicular y austenita retenida. Esto depende de la temperatura y tiempo de tratamiento isotérmico empleado.

El elevado contenido de silicio en las fundiciones nodulares evita la formación de carburos que son normales en las transformaciones bainíticas de los aceros ${ }^{[4]}$. La bainita en la fundición nodular permite alcanzar elevados valores de fractotenacidad, 60-90 MPa $\cdot \mathrm{m}^{0,5}$, resistencia a la tracción, 1.000-1.500 MPa, ductilidad 2-15\%, y elevada resistencia al desgaste ${ }^{[5]}$.

Debido a las excelentes propiedades mecánicas encontradas en las fundiciones nodulares ADI, su aplicación se ha extendido a componentes industriales sometidos a crecientes exigencias mecánicas y medio ambiente. Tales son los ejemplos del uso en cigüeñales, tambores de motores, árboles de leva, ruedas de vagones, engranajes y, eventualmente, en bolas para molinos en la minería. Estas aplicaciones requerirán de la mejora de la resistencia a la fatiga y corrosión del material.

El objetivo de este trabajo es sistematizar y orientar las investigaciones con el propósito de avanzar en el entendimiento de las relaciones entre la microestructura y las propiedades mecánicas de las fundiciones nodulares. En esta revisión se muestran los avances realizados en las investigaciones sobre las fundiciones nodulares en general, con énfasis de las austemperizadas ADI. Estas investigaciones se pueden dividir fundamentalmente en seis áreas: los procesos de solidificación, los aleantes, los tratamientos térmicos, la determinación de las propiedades mecánicas, la caracterización microestructural y fractográfica y, finalmente, la modelización numérica de las propiedades mecánicas. Algunas investigaciones incluyen una combinación de áreas, como por ejemplo el efecto de los tratamientos térmicos en las propiedades mecánicas.

\section{PROCESOS DE SOLIDIFICACIÓN}

La estructura de solidificación de las fundiciones puede ser caracterizada por el tamaño y distribución de los nódulos y las células eutécticas, la microsegregación de elementos solutos y la microes- tructura de la matriz. Solamente la matriz puede cambiarse por medio de tratamientos térmicos posteriores. La nodularidad es uno de los parámetros importantes a controlar en el proceso de solidificación ${ }^{[2]}$. Se ha encontrado que el tamaño de la célula eutéctica y la microsegregación influyen fuertemente en las propiedades mecánicas finales de la fundición ${ }^{[6]}$. Se ha normalizado la obtención de la aleación por medio de la standard de la ASTM A $897 \mathrm{M}^{[7]}$. Los últimos estudios se han concentrado en determinar el mecanismo de solidificación ${ }^{[8]} \mathrm{y}$ de grafitización ${ }^{[9-12]}$. Además, se ha avanzado en la determinación de los diagramas de solidificación $\mathrm{Fe}-\mathrm{C}-\mathrm{Si}$ por medio de análisis térmico diferencial $^{[13]}$.

\section{ELEMENTOS ALEANTES}

Las fundiciones nodulares son aleaciones base hierro en las que además de carbono y silicio están presentes en forma básica el manganeso, fósforo y azufre. Los aleantes de magnesio y cerio se ajustan para regular la nodularidad del carbono (grafito). El efecto de los inoculantes ha sido tratado ampliamente ${ }^{\left[14^{15} 15\right]}$. Los factores que afectan la distribución de los nódulos han sido revisados recientemente ${ }^{[16]}$. El efecto de los elementos aleantes en las transformaciones de fases en estado sólido ha sido tratado por numerosos investigadores. Se ha tratado, particularmente, el efecto del molibdeno, cobre y níquel en la formación de la perlita y la martensita $^{[17]}$ y en la bainita ${ }^{[18}$ y 19] . El efecto de los elementos aleantes sobre las propiedades mecánicas también ha recibido atención, habiendo sido particularmente estudiado en fundiciones nodulares ADI, el efecto del silicio en la resistencia a la tracción e impacto ${ }^{[20]}$. En fundiciones nodulares ferríticas se ha estudiado el efecto del silicio en la resistencia a la tracción, impacto ${ }^{[21]}$ y fractotenaci$\operatorname{dad}^{[22]}$.

\section{TRATAMIENTOS TÉRMICOS}

En las fundiciones nodulares la matriz puede ser cambiada por tratamientos térmicos. Las características de las transformaciones de fases en estado sólido en fundiciones nodulares han sido referidas ampliamente $\mathrm{f}^{[2 \text { 23] }}$. El recocido para obtener una matriz ferrítica es un tratamiento térmico común. Se pueden obtener diferentes proporciones de ferrita y perlita controlando la temperatura de recocido y la velocidad de enfriamiento. Por medio de la austenización seguida por temple en aceite se puede 
obtener una estructura martensítica, que puede, subsecuentemente, ser sometida a un revenido.

Durante las dos últimas décadas se ha extendido la aplicación del tratamiento de temple bainítico a las fundiciones nodulares ${ }^{[24-31]}$. También se ha avanzado en la obtención de las matrices ferríticas, a partir de la estructura de bruto de colada incrementando el contenido de silicio ${ }^{[21]}$ y ADI por medio de desmoldeo en caliente ${ }^{[4]}$.

\section{PROPIEDADES MECÁNICAS}

La caracterización de las propiedades mecánicas clásicas de las fundiciones nodulares ha recibido gran atención desde la aparición de esta aleación. En particular, se han realizados estudios sobre la resis-

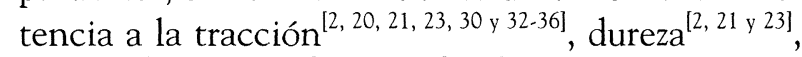
impacto $^{[2,20,21,23 \text { y } 32]}$ y fatiga ${ }^{[36-44]}$. Además, se ha estudiado el efecto del medio ambiente en las propiedades mecánicas ${ }^{[4]}$. También se ha avanzado en la determinación de las propiedades fractomecánicas del factor crítico de intensidad de esfuerzo estático, $K_{\mathrm{Ic}}^{[1,5 \text { y } 46-59]}$, dinámico, $K_{\mathrm{Id}}{ }^{[46,50 \text { y 60] }}$, y propagación de grietas de fatiga ${ }^{[42 \text { y } 61-66]}$. Según el conocimiento del autor, la caracterización de las propiedades fractomecánicas en ambiente corrosivo no ha sido investigada.

\section{CARACTERIZACIÓN MICROESTRUCTURAL Y FRACTOGRÁFICA}

Las investigaciones se han apoyado en la microscopía cualitativa y cuantitativa para la identificación de las fases y microconstituyentes obtenidos durante la solidificación ${ }^{[67-69]}$, por tratamientos térmicos clásicos ${ }^{[11,50,55 \text { y 56] }}$ y por austemperizado a diferentes temperaturas y tiempos de tratamiento térmico $^{[11,31,33,40,70 \text { y } 71]}$. Además se ha avanzado en la caracterización del tamaño y distribución de las células eutécticas ${ }^{[16]}$. Los estudios fractográficos sobre las superficies de fractura de fundiciones nodulares son, generalmente, una ampliación de la caracterización de las propiedades mecánicas de fractotenacidad ${ }^{[50,55 \text { y } 56]}$ y fatiga ${ }^{[66]}$. Solo en escasos trabajos se trata de forma exclusiva el tema fractográfico en este material ${ }^{[72]}$.

\section{MODELIZACIÓN DE LAS PROPIEDADES MECÁNICAS}

La modelización numérica de las propiedades mecánicas de las fundiciones nodulares ha recibido escasa atención. Esto puede deberse a la falta de estudios generalizados que relacionan la microestructura con las propiedades mecánicas y los micromecanismos de fractura. Son significativos los estudios de la predicción del umbral de propagación de grietas de fatiga ${ }^{[64]}$, el análisis de los micromecanismos de fatiga ${ }^{[7]}$ y la modelización numérica por elementos finitos de la resistencia a la fractura de las fundiciones nodulares ${ }^{[74]}$.

\section{DISCUSIÓN}

El efecto de la microestructura sobre las propiedades mecánicas clásicas de la resistencia a la tracción, impacto y fatiga ha sido estudiado por numerosos autores. En general, se ha encontrado que el aumento del carbón equivalente reduce las propiedades mecánicas. Además, se ha asociado un aumento en la dureza de la matriz con una mejora de las propiedades mecánicas. El caso más importante lo muestran las fundiciones nodulares ADI.

En las fundiciones nodulares el grafito se comporta como una fase con nula resistencia, dando lugar a cavidades o grietas superficiales e internas. Estas facilitan la nucleación y crecimiento de grietas bajo la acción de esfuerzos. Por lo tanto se deben considerar las propiedades fractomecánicas que evalúan la resistencia al crecimiento inestable y estable de grietas. El efecto de la microestructura sobre las propiedades fractomecánicas del $K_{\text {Ic }}$ en fundiciones nodulares ha sido estudiado por numerosos autores. Sin embargo, no se han encontrado tendencias entre los parámetros microestructurales y la fractotenacidad, lo cual difiere de estudios en fundiciones con grafito laminar ${ }^{[75]}$, en los que se observa que, para matrices de baja dureza, existe una relación de directa proporcionalidad con el KIc y para matrices con alta dureza la relación se invierte. Esto se explica en términos de los mecanismos de fractura de la matriz, que cambian de dúctil-fibroso a frágil-intergranular.

En las fundiciones nodulares se ha estudiado el efecto de los elementos aleantes y de la distribución de los nódulos de grafito en el $K_{\mathrm{Ic}}$. Además se ha investigado la fractotenacidad de fundiciones nodulares con matrices austeníticas, ferríticas, perlíticas y bainíticas con austenita retenida. Para contenidos superiores al $30 \%$ de austenita retenida se ha observado la transformación a martensita, inducida por plasticidad, durante el crecimiento inestable de grietas ${ }^{[46]}$.

El crecimiento estable de grietas ocurre por esfuerzos cíclicos de fatiga. El efecto de la microestructura sobre la resistencia al crecimiento de grietas de fatiga en fundiciones nodulares, particularmente 
$\mathrm{ADI}$, ha recibido una creciente atención ${ }^{[62-64]}$. Esto facilitará la formulación de tendencias en el comportamiento mecánico de los materiales. Los estudios sobre el efecto de la microestructura de la matriz en la propagación de grietas de fatiga han cubierto las matrices ferríticas, perlíticas, bainíticas con austenita retenida y martensíticas. Se ha encontrado que la velocidad de crecimiento de grietas se incrementa al aumentar la resistencia a la tracción de la fundición nodular y al aumentar la razón de esfuerzo cíclico ${ }^{[62]}$. En muchos casos, el efecto de estas variables desaparece si los datos de propagación de grietas de fatiga son analizados en términos del factor efectivo de intensidad de esfuerzo, $K_{\mathrm{ef}}{ }^{[62]}$. Este parámetro incluye el efecto del cierre de la grieta durante la aplicación de la carga cíclica.

Se ha propuesto un modelo microestructural basado en la teoría de dislocaciones para predecir el umbral del factor de intensidad de esfuerzos cíclicos, $K_{\text {umbral }}$. Desgraciadamente, se encuentran dificultades para aplicar este modelo a las fundiciones ADI debido a que se requieren parámetros de propiedades mecánicas que no se encuentran disponibles en la literatura. Se han encontrado valores experimentales para el $K_{u m b r a l}$, para fundiciones nodulares ADI con austenita retenida, en el rango de 4 a $7 \mathrm{MPa} \cdot \mathrm{m}^{0,5}[64]$.

\section{FUTURAS INVESTIGACIONES}

El uso intensivo de los materiales resistentes requiere un permanente avance del conocimiento sobre la relación entre la microestructura y las propiedades mecánicas. Esto se relaciona con el aumento de la capacidad del material para someterse a condiciones de servicio cada vez más exigentes a fin de ampliar su uso.

La revisión anterior demostró que faltan estudios sobre la resistencia al crecimiento de grietas bajo un ambiente agresivo de corrosión. Esto permitirá aumentar la competitividad de las fundiciones nodulares con los aceros de alta resistencia, particularmente las austemperizadas ADI, y explorar su uso en componentes mineros y navales. En estos estudios se pueden aplicar las técnicas experimentales ya desarrolladas en otros materiales ${ }^{[76-78]}$. Las variables mecánicas a considerar son: la razón de esfuerzo y la frecuencia con la forma de la onda de la carga. Las variables electroquímicas a considerar son: el potencial eléctrico, la temperatura, el $\mathrm{pH}$ y el contenido de oxígeno disuelto en el medio agresivo. Además, se requiere estudiar el efecto de las variables microestructurales consistentes en la estructura de la matriz y la célula eutéctica. En particular, se debe considerar el efecto de la austenita retenida y el tamaño de la célula eutéctica.

La propagación de grietas de fatiga bajo corrosión puede ser afectada por la corrosión bajo tensión. Por lo tanto se requiere además, caracterizar el factor crítico de esfuerzo en corrosión bajo tensión, $K_{\text {ISCC }}$, en las fundiciones nodulares austemperizadas ADI, usando las técnicas aplicadas en otros materiales ${ }^{[76]}$. Las investigaciones propuestas anteriormente están siendo desarrolladas por parte de los autores y constituyen la continuación de trabajos en fundiciones con grafito laminar ${ }^{[75 \text { y } 79]}$ y nodular ${ }^{[11 \text { y } 67]}$.

En el área de la modelización se propone extender los modelos microestructurales de la fractotenacidad desarrollados en fundiciones grises ${ }^{[75,80}$ y 81] a fundiciones nodulares. Además, los modelos microestructurales de propagación de grietas de fatiga desarrollados para fundiciones grises ${ }^{[79]}$ se pueden extender a las fundiciones nodulares. Los estudios microestructurales y fractográficos cualitativos y cuantitativos también contribuirán a determinar los micromecanismos de fractura. Para esto se pueden ampliar los estudios realizados en fundiciones grises $^{[82 \text { y } 83]}$ a las fundiciones nodulares.

\section{CONCLUSIONES}

La revisión bibliográfica ha mostrado que las fundiciones nodulares han recibido creciente atención en numerosas áreas. Esto se ha debido a la elevada resistencia y ductilidad. Considerable interés han despertado las propiedades obtenidas en fundiciones nodulares con tratamientos térmicos de temple bainítico, ADI, debido a sus excelentes propiedades mecánicas, llegando a ser comparables con los aceros.

Con el propósito de ampliar la aplicación de estos materiales a condiciones de servicio que incluyan corrosión se hace necesario avanzar en el entendimiento de la relación entre la microestructura y la resistencia al crecimiento de grietas de fatiga bajo corrosión. Además, se requiere realizar progresos en la modelización de las propiedades mecánicas y determinar los micromecanismos de fractura.

\section{Agradecimientos}

Este trabajo ha sido desarrollado en el marco de un proyecto de investigación realizado por la Universidad de La Serena y Santiago de Chile conjuntamente con la Universidad de Mar del Plata de Argentina. La financiación fue aportada por el Fondecyt por medio del proyecto 1960401. 


\section{REFERENCIAS}

[1] W.L. BRADLEY, AFS Trans. 89 (1981) 837-848.

[2] H.E. Henderson, Gray, Ductile and Malleable Iron Castings-Current Capabilities, ASTM STP 455, ASTM, Philadelphia, PA, EE.UU., 1969, pp. 29-53.

[3] T.S. SHIH, P.Y. LIN, C.H. CHANG y C.R. LOPER JR., AFS Trans., 98 (1990) 142-147.

[4] J. SCHISSLER, Proc. 3rd. International Symposium on the Physical Metallurgy of Cast Iron, Royal Institute of Technology, Estocolmo, Suecia 1984, Mat. Res. Soc. Symp. Proc., vol. 34, North Holland, New York, NY, EE.UU., 1985, pp. 335-344.

[5] J. ArANZÁbal, I. GUTIÉRREZ, J.M. RodríGUez y J.J. URCOLA, J. Mater. Sci. Tech. 8 (1992) 263-273.

[6] R. BOERI Y F. WEINBERG, AFS Trans. 97 (1989) 106-110.

[7] ASTM, Annual Book of ASTM Standards, ASTM, vol. 01.02, Philadelphia, PA., EE.UU., 1990, pp. 563-568.

[8] P.K. BASUTKARE, C.R. LOPER y C.L. BABU, AFS Trans. 78 (1970) 262-280.

[9] S.V. SUbRAMANIAN, D.A.R. KAY y G.R. PURDY, AFS Trans. 90 (1982) 589-603.

[10] L. QIFU y L. QIYANG, AFS Trans. 101 (1993) 101-109.

[11] V.M. BERMONT Y J.A. SIKORA, Int. J. Cast Metals Res. 11 (1998) 51-61.

[12] T. Skaland y O. Grong, Met. Trans. 24A (1993) 23472353.

[13] R. STARAL, B.D. JOHNSON, M.D. ROTHWELL, F.R. KAEZMARCK y R.W. HEINE, AFS Trans. 106 (1998) 65-75.

[14] M.I. Onsolen, O. Grong, T. Skaland y S.O. Olsen, AFS Trans., 105 (1997) 147-152.

[15] S.L. LIU, C.R. LOPER JR. y T.H. WITTER, AFS Trans. 100 (1992) 899-906.

[16] G.M. GOODRICH y D.P. JONES, AFS Trans. 101 (1993) 1031-1037.

[17] S.K. YU Y C. LOPER, AFS Trans. 96 (1988) 811-822.

[18] S.E. STENFORS, Proc. 3rd. International Symposium on the Physical Metallurgy on Cast Iron, Royal Institute of Technology, Estocolmo, Suecia, 1984, Mat. Res. Soc. Symp. Proc., vol. 34, North Holland, New York, NY, EE.UU., 1985, pp. 423-432.

[19] B.V. Kovacs, Proc. 3rd. World Conference on Austempered Ductile Iron, AFS, Chicago, IL, EE.UU., 1991, pp. 243-248.

[20] H. NieswaAg y J.W. Niuhof Proc. 3rd. International Symposium on the Physical Metallurgy on Cast Iron, Royal Institute of Technology, Estocolmo, Suecia, 1984, Mat. Res. Soc. Symp. Proc., vol. 34, North Holland, New York, NY, EE.UU., 1985, pp. 411-422.

[21] E.N. PAN, W.S. HSU y C.R. LOPER JR., AFS Trans. 96 (1988) 645-660.

[22] S. Komatsu y T. SHIOTA, Proc. 3rd. International Symposium on the Physical Metallurgy on Cast Iron,
Royal Institute of Technology, Estocolmo, Suecia, 1984, Mat. Res. Soc. Symp. Proc., vol. 34, North Holland, New York, NY, EE.UU., 1985, pp. 517-526.

[23] H.T. ANGus, Cast Iron: Physical and Engineering Properties, Butterworths, 2nd. ed., Londres, Inglaterra, 1976, pp. 81-82.

[24] N. DaRWISH y R. EயIOT, J. Mater. Sci. Tech. 9 (1993) 572-585.

[25] N. DarWISH y R. EmIOT, J. Mater. Sci. Tech. 9 (1993) 586-602.

[26] J. Aranzábal, I. GutiérRez y J.J. Urcola, J. Mater. Sci. Tech. 10 (1994) 728-737.

[27] T.N. Rouns y K.B. RUNDMAN, AFS Trans. 95 (1987) 851-874.

[28] J.M. MASSONE, R.E. BOERI Y J.A. SIKORA, AFS Trans. 104 (1996) 133-137.

[29] R.C. VoIGt y C.R. LOPER JR., Proc. 3rd. International Symposium on the Physical Metallurgy on Cast Iron. Royal Institute of Technology, Estocolmo, Suecia, 1984, Mat. Res. Soc. Symp. Proc., vol. 34, North Holland, New York, NY, EE.UU., 1985, pp. 377-386.

[30] R.B. GUNDLACH, J.F. JANOWAK, S. BECHET Y K. ROHRIG, Proc. 3rd. International Symposium on the Physical Metallurgy on Cast Iron. Royal Institute of Technology, Estocolmo, Suecia, 1984, Mat. Res. Soc. Symp. Proc., vol. 34, North Holland, New York, NY, EE.UU., 1985, pp. 399-409.

[31] B. KOVACS, AFS Trans. 99 (1991) 283-285.

[32] T.S. SHIH, C.K. LIN, y H.Z. TwaN, AFS Trans. 105 (1997) 367-376.

[33] T.N. Rouns, K.B. Rundman y D.M. MOORE, AFS Trans. 92 (1984) 815-840.

[34] G.P. FAubert, D.J. MOORE y K.B. Rundman, AFS Trans. 98 (1990) 759-764.

[35] N. DarWISH y R. EmIOT, J. Mater. Sci. Tech. 9 (1993) 882-889.

[36] K.L. HAYRYNeN, D.J. MOORE y K.B. RUNDMAN, AFS Trans. 100 (1992) 93-104.

[37] G.P. FAUBERT, D.J. MOORE y K.B. RUNDMAN, AFS Trans. 99 (1991) 563-570.

[38] D. Venugopalan y A. Alagarsamy, AFS Trans. 100 (1992) 337-341.

[39] D. Venugopalan y K.L. Pilon, AFS Trans. 96 (1988) 697-704.

[40] J.F. Janowak y A. Alargarsamy, AFS Trans. 98 (1990) 511-518.

[41] M. SOFUE, S. OKADA y T. SASAKI, AFS Trans. 86 (1978) 173-182.

[42] M.S. StarkeY y P.E. IRVING, Int. J. Fatigue (1982) 129136.

[43] A.G. FULLER, AFS Trans. 85 (1977) 527-536.

[44] B. Ostensson, J. Iron Steel Inst. London 211 (1973) 628-631. 
[45] S. Muthukumarasamy y S. Seshan, AFS Trans. 100 (1992) 873-879.

[46] J. AranzÁbal, I. GutiérRez, J.M. Rodríguez y J.J. URCOLA, Anales de Mecánica de la Fractura 7 (1990) 39-46.

[47] W.L. Bradley, K.E. MCKININEY y P.C. GerHardt JR., Fracture Mechanics: Seventeenth Volume, ASTM STP 905, ASTM, Philadelphia, PA, EE.UU., 1986, pp. 75-94.

[48] S. NishI, T. KobAYASH y S. TAGA, J. Mater. Sci. 11 (1976) 723-730.

[49] A. Lazaridis, C.R. Loper y R.W. Heine, AFS Trans. 79 (1971) 351-360.

[50] H.E. MEAD JR. y W.L. BRAdLEY, AFS Trans. 88 (1980) 265-277.

[51] I.L. MOgford, I.L. Brown y D. HuLL, J. Iron Steel Inst. London 205 (1967) 729-732.

[52] S.C. LeE y C.C. LeE, AFS Trans. 96 (1988) 827-838.

[53] J.L. Doong y C.S. CHEN, J. Fatigue Fract. Engng. Mater. Struct. 12 (1989) 155-165.

[54] J. NiskaneN, AFS International Cast Metals J. 2 (1977) 20-22.

[55] R.K. NANSTAD, F.J. WORZAlA y C.R. LOPER JR., AFS Trans. 82 (1974) 473-486.

[56] R.K. NANSTAD, F.J. WORZOLA y C.R. LOPER JR., AFS Trans. 83 (1975) 245-256.

[57] S.R. HOLDSWORTH y G. JOLLEY, J. British Foundryman 68 (1975) 169-172.

[58] E. DorazIl, M. Holzmann, Proc. 3rd. World Conference on Austempered Ductile Iron, AFS, Chicago, IL, EE.UU., 1991, pp. 32-66.

[59] T. Kobayashi, H. Yamamoto y S. Yamada, Proc. 3rd. World Conference on Austempered Ductile Iron, AFS. Chicago, IL, EE.UU., 1991, pp. 567-576.

[60] B. Faucher, K.C. Wang y M. Gagne, AFS Trans. 95 (1987) 127-132.

161] F.J. Belzunce y C. Rodríguez, Anales de Mecánica de la Fractura 7 (1990) 47-52.

[62] K. Tokal, T. Ogawa y K. Shamoto, Int. J. Fatigue 16 (1994) 344-350.

[63] B. Kovacs, AFS Trans. 100 (1992) 130-134.

[64] L. Bartosiewiez, A.R. Krause, B. Kovacs y S.K. PUTATUNDA, AFS Trans. 100 (1992) 135-142.

[65] D.J. BuLlOCH y J.H. BuLlOCH, Int. J. Pres. Ves. Piping 36 (1989) 289-314.
[66] M. Castagna, P. Ferrero y R. Medana, AFS Trans. 86 (1978) 573-582.

[67] J. A. SikORA, G. L. Rivera y H. Biloni, Proc. International Symposium on Solidification, Pergamon Press, New York, NY, EE.UU., 1990, pp. 280-285.

[68] G.L. RIVERA, R.E. BOERI y J.A. SIKORA, Int. J. Cast Metals Res. 8 (1995) 1-5.

[69] R. VOIGT y C. LOPER, AFS Trans. 97 (1989) 595-600.

[70] D. KRISHNARA, H.N.L. NarasimHan y S. SeShan, AFS Trans. 100 (1992) 105-112.

[71] J. Aranzábal, I. GutiérRez y J.J. Urcola, J. Mater. Sci. Tech. 10 (1994) 728-737.

[72] N. LAZARDIS, R.K. NANSTAD, F.J. WORZALA y C.R. LOPER JR., AFS Trans. 85 (1977) 277-288.

[73] W.J. VILLACIS y J.A. SIKORA, Int. J. Cast Metals Res. 1 (1989) 210-215.

[74] M.F. BRERETON y A.J. PADKIN, Internal Report N.84/A.B1 on the Effect of Microstructure on Fatigue Crack Growth, University of Bristol, Department of Mechanical Engineering, Bristol, Inglaterra, 1984, pp. 12-17

[75] R.N. CASTILO Y T.J. BAKER, Proc. 3rd. International Symposium on the Physical Metallurgy of Cast Iron. Royal Institute of Technology, Estocolmo, Suecia, 1984, Mat. Res. Soc. Symp. Proc., vol. 34, North Holland, New York, NY, EE.UU., 1985, pp. 487-495.

[76] I. LemaY, Priciples of Mechanical Metallurgy, Elsevier North Holland, INC, 1st. ed., New York, NY, EE.UU., 1981, pp. 291-295; 328-330.

[77] ASTM, Annual Book of ASTM Standards, ASTM E647, ASTM, vol.10, Philadelphia, PA, EE.UU., 1978.

[78] Annual Book of ASTM Standards, ASTM E742, ASTM. vol. 10, Philadelphia, PA, EE.UU., 1981.

[79] R.N. CASTILLO y T.J. BAKER, Proc. 6th International Conference on Fracture, vol. 3, National Aeronautical Lab., New Delhi, India, Ed. S. R. Valluri, Pergamon Press, New York, NY, EE.UU., 1984.

[80] E. HORnBogen y J.M. Motz, AFS Int.Cast Metals J. 2 (1977) 31-36.

[81] D.K. VERMA y J.T. BERRY, AFS Trans. 89 (1981) 849856.

[82] L. HaENny y G. Zambell, AFS Trans. 90 (1982) 275-283.

[83] R.C. VoIGt y S.D. HOLmGREN, AFS Trans. 98 (1990) 213-225. 